\title{
ç \\ Efficient generation of short and high-power x-ray free-electron-laser pulses based on superradiance with a transversely tilted beam
}

\author{
Eduard Prat, Florian Löhl, and Sven Reiche \\ Paul Scherrer Institut, CH-5232 Villigen PSI, Switzerland \\ (Received 22 July 2015; published 12 October 2015)
}

\begin{abstract}
$\mathrm{X}$-ray free electron lasers (XFELs) are innovative research tools able to produce high-power and short radiation pulses for multiple scientific applications. We present a new method to produce XFEL radiation with much higher power and shorter pulse lengths than the ones obtained at standard XFEL facilities. This will enable new kinds of experiments in scientific fields such as nonlinear optics and bioimaging. The scheme is based on introducing a transverse tilt to the electron beam, thus limiting the fraction of the bunch able to produce XFEL radiation. In the first part of the undulator beam line only the tail of the electron bunch lases. Then, by properly delaying and correcting the trajectory of the electron beam between some undulator modules, all the electrons can contribute to the amplification of a very short XFEL pulse. Apart from being efficient, our method is flexible since by tuning the tilt amplitude one can obtain shorter or more energetic XFEL pulses. The scheme can readily be applied since, besides the standard components of an XFEL facility, it only needs small chicanes between certain undulator modules. We have confirmed the validity of our proposal with numerical simulations done for the SwissFEL case.
\end{abstract}

DOI: 10.1103/PhysRevSTAB.18.100701

PACS numbers: 41.60.Cr, 29.20.Ej, 42.55.Vc

\section{INTRODUCTION}

X-ray free electron lasers (XFELs) are cutting-edge scientific instruments in various research fields such as biology, material science, chemistry, and physics. Most XFELs are based on the self-amplified spontaneous emission (SASE) process [1,2], though self-seeding allows improving the longitudinal coherence of the SASEXFELs for both the soft and the hard $\mathrm{x}$-ray regime [3-6]. State-of-the-art XFELs generate almost fully coherent radiation with pulse powers of a few tens of gigawatts and pulse durations of several tens of femtoseconds and shorter $[7,8]$. There is, however, a strong desire in research areas such as nonlinear optics and bioimaging to achieve even shorter pulses and/or higher radiation powers-see for example Refs. [9-15].

There are several proposals to shorten the XFEL pulses below the femtosecond level keeping an equivalent radiation power level as in standard facilities, either by reducing the electron pulse length $[16,17]$ or by using external lasers [18-24]. In 2013 Tanaka proposed a scheme to generate attosecond pulses with radiation powers in the terawatt level [25]. More recently we presented a simple and feasible method to achieve terawatt-attosecond XFEL pulses [26]. These two latter proposals share, however, the inconvenience that the electron bunch is not used very

*eduard.prat@psi.ch

Published by the American Physical Society under the terms of the Creative Commons Attribution 3.0 License. Further distribution of this work must maintain attribution to the author $(s)$ and the published article's title, journal citation, and DOI. efficiently: in the scheme of Ref. [25] the use of an emittance spoiler [16] and the enhanced-SASE technique [20] limits significantly the number of electrons contributing to the lasing process, and in our case [26] the emittance spoiler entails that only about $20 \%$ of the electron beam contribute to the generation of the XFEL pulses.

Here we present a new method to generate short and high-power XFEL pulses in an efficient and flexible way: practically $100 \%$ of the electrons can contribute to the XFEL process and the scheme can be tuned for minimum pulse length or maximum pulse energy. The method is based on superradiance [27,28] and the use of a transversely tilted beam. Emma and Huang already proposed to tilt the beam to reduce the XFEL pulse duration [29]. Here we extend their idea to generate shorter and high-power XFEL pulses: by suitably correcting the trajectory and delaying a tilted electron beam between certain undulator sections all the electrons can contribute to enhance a short XFEL pulse in the superradiance regime. Besides the standard elements of a typical XFEL facility, the scheme only needs small magnetic chicanes between certain undulator modules. The transverse tilt can be achieved with standard procedures (see below). Therefore, our scheme is feasible and can be implemented in any of the existing or future XFEL facilities with modest hardware modifications.

\section{DESCRIPTION OF THE SCHEME}

The scheme is physically based on: (i) the superradiant regime $[27,28]$, in which there is a shortening of the XFEL pulse length and an increase of the pulse energy at the same 
time; (ii) the stringent requirements on the trajectory of the electron beam for the XFEL process, since the amplification process of the XFEL pulses requires a good transverse overlap between the electron and photon beams.

\section{A. Layout and working principle}

Figure 1 shows a layout of the proposed scheme. An undulator section consists of one or more undulator modules. In the first part before the undulator beam line the electron beam is transversely tilted, i.e., a monotonic correlation between the trajectory and the longitudinal position of the bunch is introduced. In Sec. II C we will discuss how to generate the transverse tilt. We will restrict ourselves to a linear transverse tilt, although in general the tilt only needs to be monotonous. We define the tilt amplitude in offset and angle as $d x / d s$ and $d x^{\prime} / d s$, respectively, where $x$ is the trajectory offset, $x^{\prime}$ is the trajectory angle, and $s$ is the longitudinal position along the bunch.

Figure 2 indicates how the scheme functions qualitatively. In the case of a tilted electron bunch, only a small region of the bunch with an adequate trajectory will produce XFEL radiation, while the rest of the bunch will not contribute to the lasing process. Let us call this part of the beam the good trajectory region (GTR). In our proposal the tail of the bunch corresponds to the GTR at the entrance of the undulator beam line. After the first undulator section formed by a few undulator modules, the produced radiation consists of a short XFEL pulse defined by the GTR. After that the electron beam is delayed such that the photon pulse overlaps longitudinally with a fresh part of the electron beam just behind the tail, a region that did not produce XFEL radiation in the previous stage. To allow also a transverse overlap, this region of the electron beam must become the new GTR of the bunch, which is achieved by modifying the electron trajectory with dipole corrector magnets. In the next undulator section the XFEL pulse will be further amplified in the superradiant regime by the new and fresh GTR, while the rest of the electrons will not contribute to the XFEL process. This procedure will be repeated until several regions of the electron beam have contributed to amplify the XFEL pulse.

The length of the GTR is related to the trajectory acceptance (TA) of the XFEL radiation, i.e., the trajectory limits for which the XFEL process can occur. The TA for a given radiation wavelength depends on the electron and undulator parameters and needs to be measured and/or

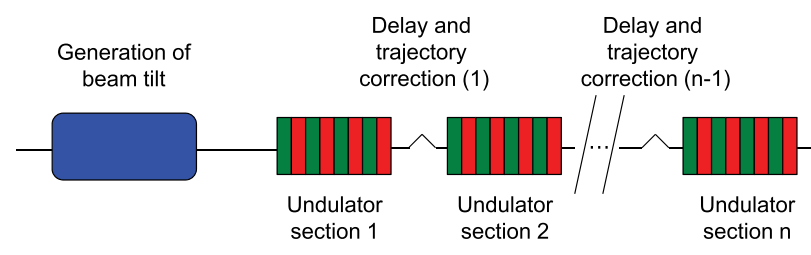

FIG. 1. Layout of the proposed scheme.

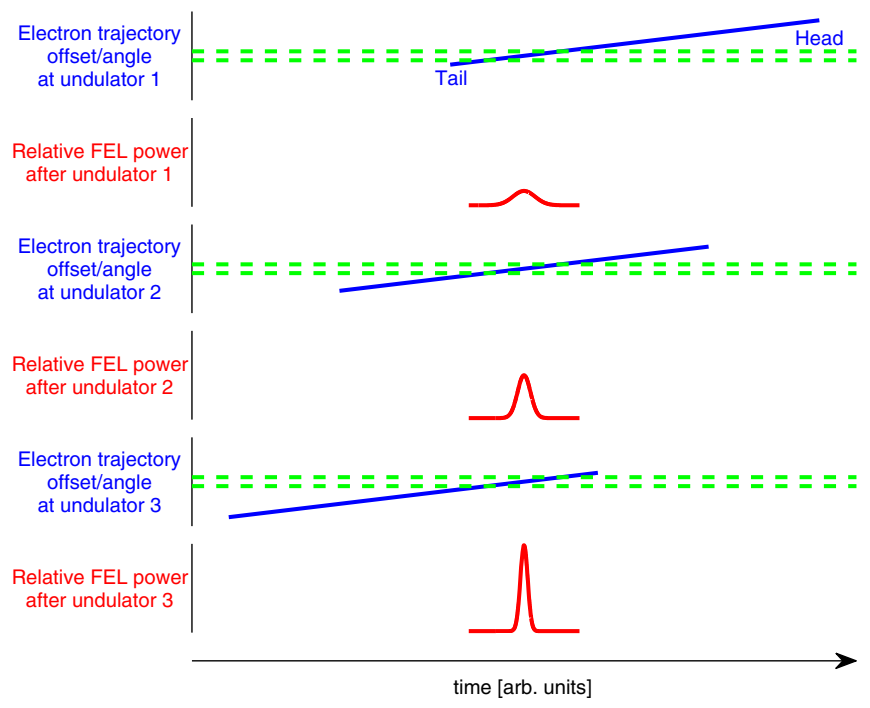

FIG. 2. Working principle of the proposed scheme for an example case with three undulator sections. The horizontal axis for all the plots is time in the reference system of the radiation field. The dashed green lines define the GTR outside of which the electron beam cannot significantly amplify the XFEL radiation. In the first undulator section only the tail of the electron beam produces XFEL radiation. After each undulator section the electron beam trajectory is corrected and the bunch is delayed such that all other regions of the electron beam contribute consecutively to enhance the XFEL pulse generated in the first undulator section.

simulated. The ratio between the TA and the tilt defines the GTR: for instance, if the TA in offset is $0.1 \mathrm{~mm}$ and the tilt amplitude in offset is $1 \mathrm{~mm}$ for the whole bunch length, the GTR will be $10 \%$ of the total bunch length. If the transverse tilt along the whole bunch length is smaller than the TA all the electrons of the bunch will produce XFEL radiation like in a normal SASE scheme.

A layout with $n$ undulator sections corresponds to $(n-1)$ locations to delay and correct the trajectory of the electrons, and to slicing the beam into $n$ parts. A certain region of the electron beam is supposed to lase at its corresponding section-for instance, if there are five undulator sections, the last $20 \%$ of the bunch should lase at the first undulator section, the next $20 \%$ at the second section, and so on. The delay between each section should be the total bunch length divided by $n$, less the slippage length along the upstream undulator section. The trajectory must be corrected such that at a certain undulator section the related electron beam region is centered.

\section{B. Performance of the scheme}

In comparison to previous proposals [25,26], this method is more efficient since all the electrons of the bunch can potentially contribute to the XFEL process. If the GTR is shorter due to a stronger tilt the beam allows for more regions. Of course it then has to be matched with the same number of undulator sections for maximum efficiency. 
In other words, if the available number of undulator sections is $n$, the most efficient use of the electron beam occurs when the GTR length is equal to the total electron bunch length divided by $n$. Let us call this optimum GTR length $L_{e}$. If the actual GTR is shorter than $L_{e}$, some of the electrons will not contribute to the XFEL process but the XFEL power will remain constant: the XFEL pulse will be shorter at the expense of a smaller pulse energy. At the other extreme, if the GTR is longer than $L_{e}$, the XFEL pulse would overlap in the second and following undulator sections with nonfresh electrons that already contributed to the XFEL process in upstream undulator sections, resulting in a degraded XFEL performance. This will result in a decrease of the XFEL peak power: although the XFEL pulse energy may increase, it will be outweighed by the associated increase in XFEL pulse length. Therefore, for a good performance the GTR length should be equal to or shorter than $L_{e}$ (the ratio between the total bunch length and the number of undulator sections).

The method is flexible since the GTR length, and therefore the XFEL performance, can be changed conveniently by tuning the tilt amplitude. In the next section we will show with numerical simulations how the XFEL peak power, pulse energy, and pulse length depend on the tilt amplitude.

For an optimum performance, the XFEL process should go into the exponential regime in the first undulator section but without entering into saturation, while the length of the following undulator sections should allow a continuous radiation growth beyond the saturation level. The number of modules for each undulator section is a parameter that needs to be optimized by numerical simulations and/or empirically. The length of a section is chosen such that with an additional module no further growth occurs. At that point the radiation is shifted to the next GTR. In the superradiant regime this occurs when the electron beam has slipped forward a distance equivalent to the separation between two spikes. Further slippage will not allow a further growth since the preceding spike has spoiled the electron beam quality.

Similar to the scheme of Ref. [26], in this proposal the wakefields along the undulator beam line can be compensated locally [30], since only a small part of the bunch lases at each undulator section. This allows using higher compression factors than in the standard operation where the full electron bunch is supposed to generate XFEL radiation, therefore requiring a global compensation to keep the entire bunch in resonance. In comparison to our recent proposal [26], this scheme is more robust against time and energy jitter since the long delays applied to a small region of the bunch are avoided here.

\section{Implementation of the scheme}

The scheme requires a transversely tilted electron bunch. The generation of the tilt can be achieved in different ways using standard components of a typical XFEL facility, as discussed previously in Ref. [29]. One possibility is to streak the beam with a transverse deflector structure [31], which is routinely used in several facilities to measure the longitudinal properties of the beam. Another option is to employ the wakefields [32] of the accelerator structures of the facility-more effective devices like corrugated pipes or dielectric structures may also be used. Moreover, the transverse tilt can be achieved by introducing dispersion to an energy-chirped beam [33]: the energy chirp can be generated by going off-crest in some accelerator structures of the XFEL facility, and/or by using the wakefields of accelerator cavities or the so-called dechirpers [34-36], while the dispersion can easily be obtained by having it leaking out from locations with strong dispersion (e.g., bunch compressors or energy collimators). Since our method works locally an energy chirp is tolerable, which otherwise blows up the bandwidth in standard SASE operation. In the case with an energy chirp, the field of the different undulator sections will have to be tuned to keep the radiation wavelength on resonance along the entire undulator beam line. An advantage of a chirped beam is that a monochromator can be used to remove the residual radiation generated at different wavelengths by other parts of the bunch at different energies.

The magnitude of the tilt needs to be measured to have full control over it and thus over the performance of the scheme. The tilt can be measured by streaking the beam in the opposite direction of the tilt-e.g., a horizontal tilt can be measured by streaking the beam vertically. The streaking can be done in the same way as the tilt was generated, for instance with a transverse deflector, using transverse wakefields or introducing dispersion to an energy-chirped beam. The tilt amplitude in offset and angle can be reconstructed by measuring the tilt amplitude in offset for different optics - this is equivalent to an emittance measurement where the beam size is measured for different optics. More information about how to measure a transverse tilt can be found in Ref. [37].

The dipole strengths required to achieve an optimum transverse overlap can be calculated from the known tilt (offset and angle) at the undulator entrance and the optics along the undulator beam line. If necessary an orbit response measurement will help to do the work. The delay to be applied by the chicanes corresponds to the bunch length divided by the number of sections, less the slippage along the undulator section. The delay is at most only a fraction of the bunch length: considering that the typical electron pulse duration is a few tens of femtoseconds, the required delays will be up to several femtoseconds. The overlap after each undulator section can be empirically optimized by adjusting the electron beam delay and trajectory while monitoring the XFEL performance (pulse length and energy).

A magnetic chicane consisting of three dipole magnets can be employed to delay the electron beam up to several 
femtoseconds. The chicanes can be designed with standard dipole magnets (with peak fields on the order of one Tesla) to be shorter than one meter, thus they can be placed in the space between undulator modules of a standard XFEL facility. If the dipoles of the chicanes have independent power supplies, the chicane can also be used to correct the trajectory of the electrons. Alternatively, the trajectory correction can be achieved with an additional pair of dipole corrector magnets.

\section{SIMULATIONS}

We have confirmed the validity of the proposed scheme with numerical calculations performed with the code Genesis 1.3 [38]. Simulations of the XFEL process with a strong tilt are challenging because the grid upon which the radiation field is evaluated must be extended by more than 1 order of magnitude, increasing the computational time by the same amount. To overcome this limitation the latest version of Genesis [39] has been used, which tracks particles but does not discard them once they are outside of the domain of the radiation grid. In this way the entire bunch can be modeled self-consistently and in a single run.

\section{A. Electron and undulator parameters}

The simulations are done for the SwissFEL project [40], presently under construction at the Paul Scherrer Institute. We have simulated the scheme for a radiation wavelength of $1 \AA$. The electron beam has the following parameters: flat current profile with a value of $6 \mathrm{kA}$ and a total charge of $200 \mathrm{pC}$, flat energy profile with a mean energy of $5.8 \mathrm{GeV}$ and a slice energy spread of $350 \mathrm{keV}$ (a flat profile is not required but it simplifies the simulations), normalized transverse emittance of $300 \mathrm{~nm}$ (consistent with our measurements at the SwissFEL Injector Test Facility [41]), average $\beta$-function in the undulator of $10 \mathrm{~m}$. The undulator modules are $4 \mathrm{~m}$ long, the undulator period is $15 \mathrm{~mm}$ and the nominal undulator parameter $K$ is 1.2 , which corresponds to a radiation wavelength of $1 \AA$ A. Table I shows the electron and undulator parameters used as input in the simulations.

TABLE I. Electron and undulator parameters for SwissFEL taken as input for the simulations.

\begin{tabular}{lc}
\hline \hline Radiation wavelength & $1 \AA$ \\
Current & $6 \mathrm{kA}$ \\
Total charge & $200 \mathrm{pC}$ \\
Energy & $5.8 \mathrm{GeV}$ \\
Energy spread & $350 \mathrm{keV}$ \\
Normalize emittance & $300 \mathrm{~nm}$ \\
Average $\beta$-function & $10 \mathrm{~m}$ \\
Undulator module length & $4 \mathrm{~m}$ \\
Undulator period & $15 \mathrm{~mm}$ \\
Undulator $K$-value & 1.2 \\
\hline \hline
\end{tabular}

The space between undulator modules is $0.75 \mathrm{~m}$. The dipole magnets of the second bunch compressor of SwissFEL have a length of $50 \mathrm{~cm}$, an aperture of $22 \mathrm{~mm}$, and can deflect a $5.8 \mathrm{GeV}$ electron beam by 1.38 degrees. Equivalent magnets could be built with $12 \mathrm{~mm}$ aperture and $5 \mathrm{~cm}$ length to provide a deflection of 0.25 degrees. A magnetic chicane of $0.45 \mathrm{~m}$ made of such magnets would be able to delay the electrons by $10 \mathrm{fs}$, which is more than sufficient in our case. The trajectory correction could be done with the first and last dipole magnets of the chicane (the correction strength is only a fraction of the required strength for the delay). A quadrupole magnet required to focus the beam could be placed in the remaining space before or after the chicane.

\section{B. Results for two different tilt amplitudes}

Considering an initial tilt only in offset in the horizontal direction, we have simulated two different cases: one with a moderate tilt amplitude in offset of $d x / d s=50$ (i.e., an initial offset of $0.5 \mathrm{~mm}$ along the whole bunch) and one with a strong tilt amplitude in offset of 100 (an initial offset of $1 \mathrm{~mm}$ ). These transverse tilts could be obtained for instance by generating dispersion with a quadrupole magnet at the second bunch compressor of SwissFEL. ${ }^{1}$

Preliminary simulations show that the GTR is a bit shorter than $10 \%$ of the total bunch length for the first case with an initial tilt amplitude in offset of 50. We used ten undulator sections for that initial tilt-in this way we are using the electron beam quite efficiently but with some margin to prevent the overlap of the XFEL radiation with degraded parts of the electron beam. For the second case (initial tilt amplitude in offset of 100) we have chosen a configuration with 20 undulator sections. The electron beam delays between the undulator sections are about $1 \mu \mathrm{m}$ for ten sections and $0.5 \mu \mathrm{m}$ for the 20 sections case. The tilt in both offset and angle evolves along the undulator following the optics of the beam line, which consists in a continuous Focus-Drift-Defocus-Drift (FODO) lattice.

\footnotetext{
${ }^{1}$ The invariant of the electron beam trajectory is $I=\gamma x^{2}+2 \alpha x x^{\prime}+\beta x^{\prime 2}$, where $\alpha, \beta$, and $\gamma$ are the Twiss parameters. A total trajectory offset of $1 \mathrm{~mm}$ along the bunch at the undulator entrance, i.e., $\pm 0.5 \mathrm{~mm}$ from the bunch center, corresponds for our beam optics to an invariant $I=67 \mathrm{~nm}$. For $\beta=25 \mathrm{~m}$ at the quadrupole position, the required trajectory kick (angle) to generate this invariant would be about $50 \mu \mathrm{rad}$. This corresponds, considering that at the second bunch compressor the electron beam has an energy chirp of about $\pm 1 \%$, to a dispersion angle of about $5 \mathrm{mrad}$ (the energy chirp is later removed using the wakefields of the accelerator structures). The required integrated normalized gradient along the quadrupole $k l$ can be calculated as the ratio between the needed dispersion angle and the dispersion offset at the magnet position. Taking into account that the dispersion at the second bunch compressor of SwissFEL is up to about $250 \mathrm{~mm}$, a dispersion angle of $5 \mathrm{mrad}$ could be achieved with a quadrupole magnet of a length $l$ of $0.1 \mathrm{~m}$ and a strength $k$ of about $0.2 \mathrm{~m}^{-2}$.
} 
After every undulator section, the centroid offset and angle are corrected to achieve a good transverse overlap between the XFEL radiation and the next fresh region of the electron beam. Figure 3 shows the trajectory offset and angle along the bunch length after every other undulator section for the case of an initial tilt amplitude in offset of 50 .

For the two cases we have optimized the number of modules to be placed in each section and the linear tapering of the undulator field [42], in the same manner as we did in Ref. [26]. For each of the configurations we have run five simulations utilizing different seeds for the generation of the electrons' shot noise. The best configuration for the undulator beam line is with four modules in the first section, with two modules in the second and third sections, and with one module in all of the following sections. The optimum taper is about $0.1 \%$ per section between the first and fifth undulator sections. For the remaining sections the undulator field is kept constant.
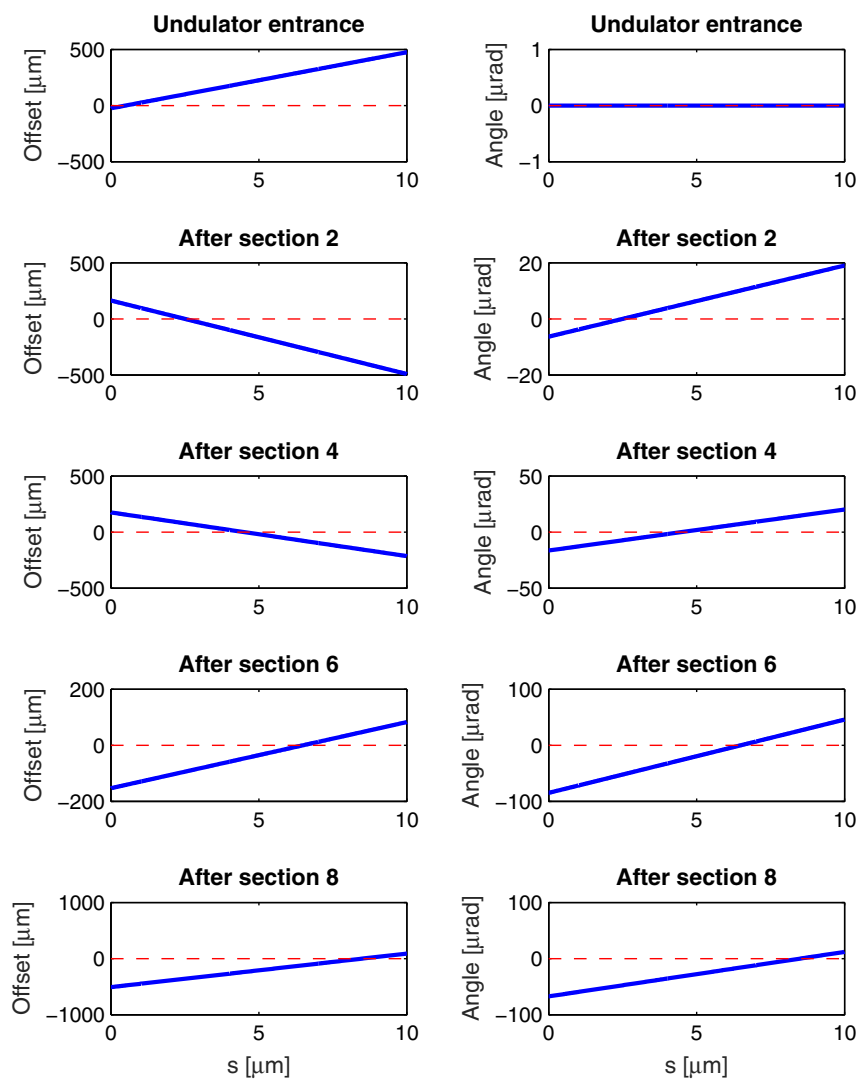

FIG. 3. Trajectory offset (left plots) and angle (right plots) as a function of the longitudinal position of the bunch at the undulator entrance (top plots) and after every other undulator section for the case of an initial tilt amplitude in offset of 50. The blue solid lines indicate the tilt and the red dashed lines show for reference the aligned trajectory. The horizontal axis corresponds to longitudinal position along the bunch in all plots. The trajectory correction at the exit of each section can be performed with the first and last dipole magnets of the magnetic chicane used also to delay the electron beam (see text for more details). The phase advance per undulator module is about 30 degrees.
For the case with the moderate tilt and ten undulator sections, the obtained final XFEL power is $0.91 \pm 0.21 \mathrm{TW}$ (the error indicates the standard deviation over the five seeds), the pulse energy is $492 \pm 24 \mu \mathrm{J}$, and the rms pulse duration is $563 \pm 17$ as. For the stronger tilt and 20 undulator sections, the final obtained XFEL power is $2.38 \pm 0.94 \mathrm{TW}$, the pulse energy is $732 \pm 41 \mu \mathrm{J}$, and the rms pulse duration is $363 \pm 6$ as. As expected, for shorter GTRs we have fewer spikes and therefore stronger fluctuations. Figure 4 shows the evolution along the undulator beam line of the XFEL pulse peak power, pulse energy, and pulse duration for all the simulated cases. As expected, one observes that, for the same undulator length,
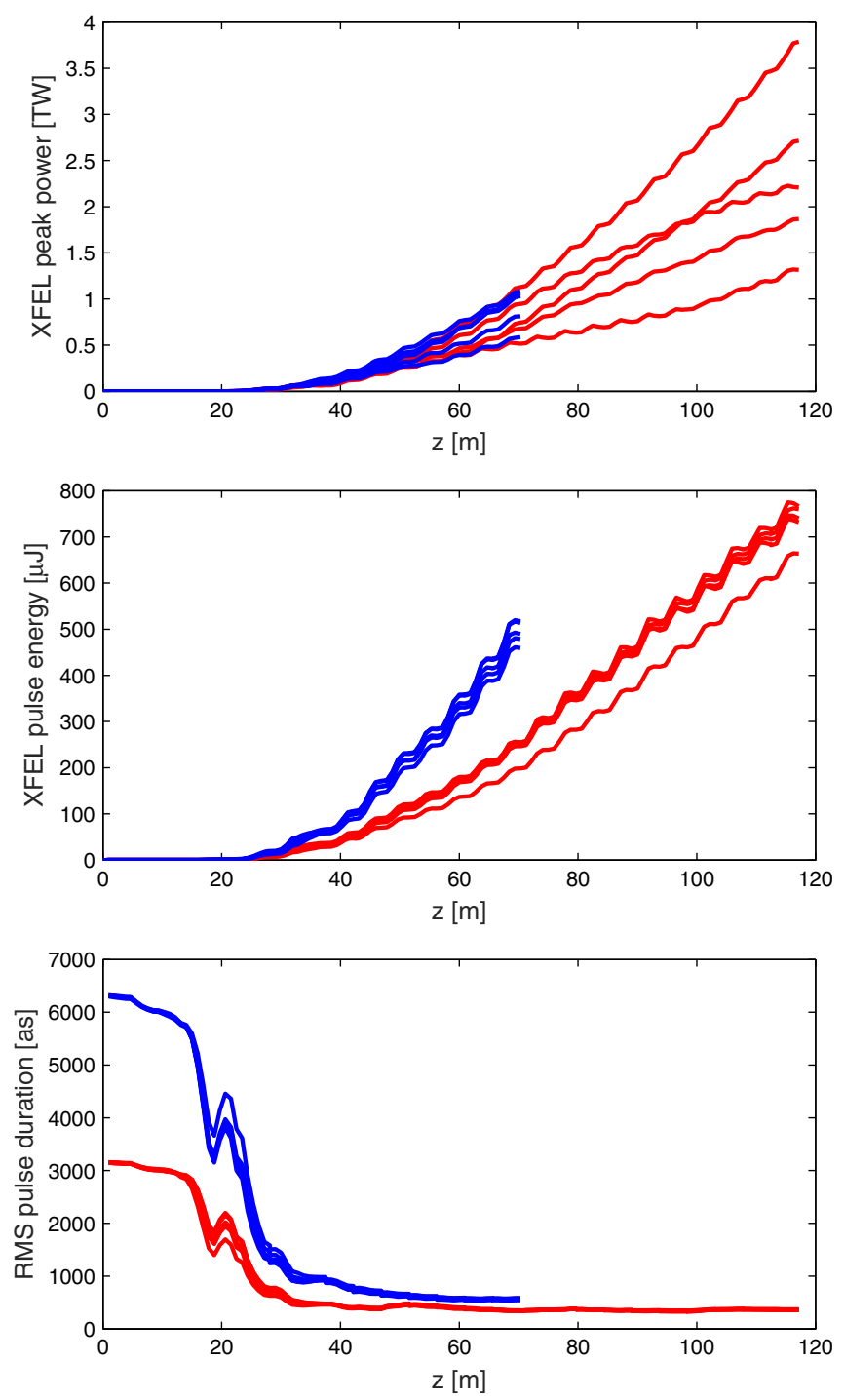

FIG. 4. XFEL peak power (top), XFEL pulse energy (center), and rms pulse duration (bottom) along the undulator beam line for the two simulated cases: initial tilt amplitude in offset of 50 with ten undulator sections (blue curves) and initial tilt amplitude in offset of 100 with 20 sections (red curves). We note that the rms pulse length is significantly affected by the spontaneous radiation background until $z \approx 30 \mathrm{~m}$. 


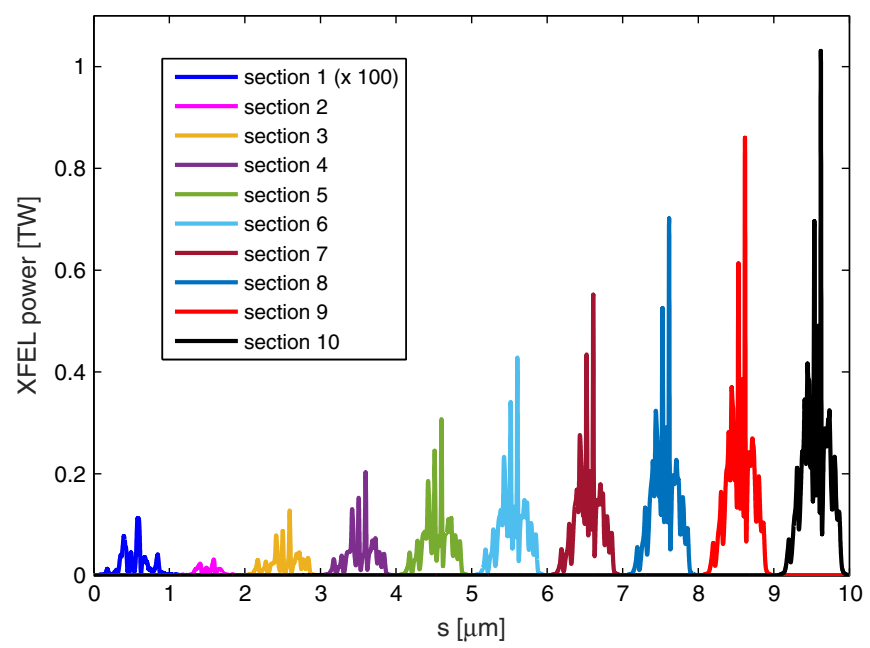

FIG. 5. XFEL peak power at $1 \AA$ along the longitudinal position of the electron bunch after each undulator section for the case with an initial tilt amplitude of 50 in offset. The final XFEL peak power is around $1 \mathrm{TW}$, the pulse energy is around $500 \mu \mathrm{J}$, and the rms pulse duration is around 500 as.

the XFEL power level is equivalent for both cases and that for the stronger tilt the XFEL pulse is shorter at the expense of a smaller XFEL pulse energy. Equivalently, for the weaker tilt the pulse energy is larger at the expense of a longer pulse. Figure 5 shows the XFEL pulse at the exit of each undulator section for one of the five shot-noise seeds corresponding to the case with the moderate tilt. Table II summarizes the simulation results for the two considered cases. As can be seen from the table and from Fig. 4, the XFEL peak power depends approximately quadratically on the undulator length, as expected from the superradiance regime $[27,28]$. This is more efficient than standard tapering with self-seeding, where the growth is only linear [43].

\section{Performance dependence on the tilt amplitude}

As explained qualitatively in the previous section, for a given undulator length a shorter GTR (obtained with a larger tilt) will give shorter XFEL pulses but lower pulse energies, while a longer GTR (obtained with a smaller tilt) will provide longer radiation pulses but with higher pulse

TABLE II. Simulation results for the two different tilt amplitudes.

\begin{tabular}{lcc}
\hline \hline & Moderate tilt & Strong tilt \\
\hline Initial tilt amplitude in offset & 50 & 100 \\
Undulator sections & 10 & 20 \\
Undulator modules & 15 & 25 \\
Total length [m] & 70 & 117 \\
Peak power [TW] & $0.91 \pm 0.21$ & $2.38 \pm 0.94$ \\
Pulse energy [ $\mu \mathrm{J}]$ & $492 \pm 24$ & $732 \pm 41$ \\
rms pulse duration [as] & $563 \pm 17$ & $363 \pm 6$ \\
\hline \hline
\end{tabular}

energies. The results for two different tilt amplitudes presented in the previous subsection confirm this.

In this subsection we systematically analyze the dependence of the XFEL performance on the tilt amplitude. For the same electron and undulator parameters shown in Table I, we have simulated how the XFEL pulse power, energy and length change with the initial tilt amplitude in offset for an undulator beam line consisting of ten sections (15 modules). We have done the simulations for one random seed for the generation of the shot noise. The results are presented in Fig. 6 and indicate that the most efficient use of the electron beam occurs for an initial tilt amplitude in offset of approximately 40 (close to the 50
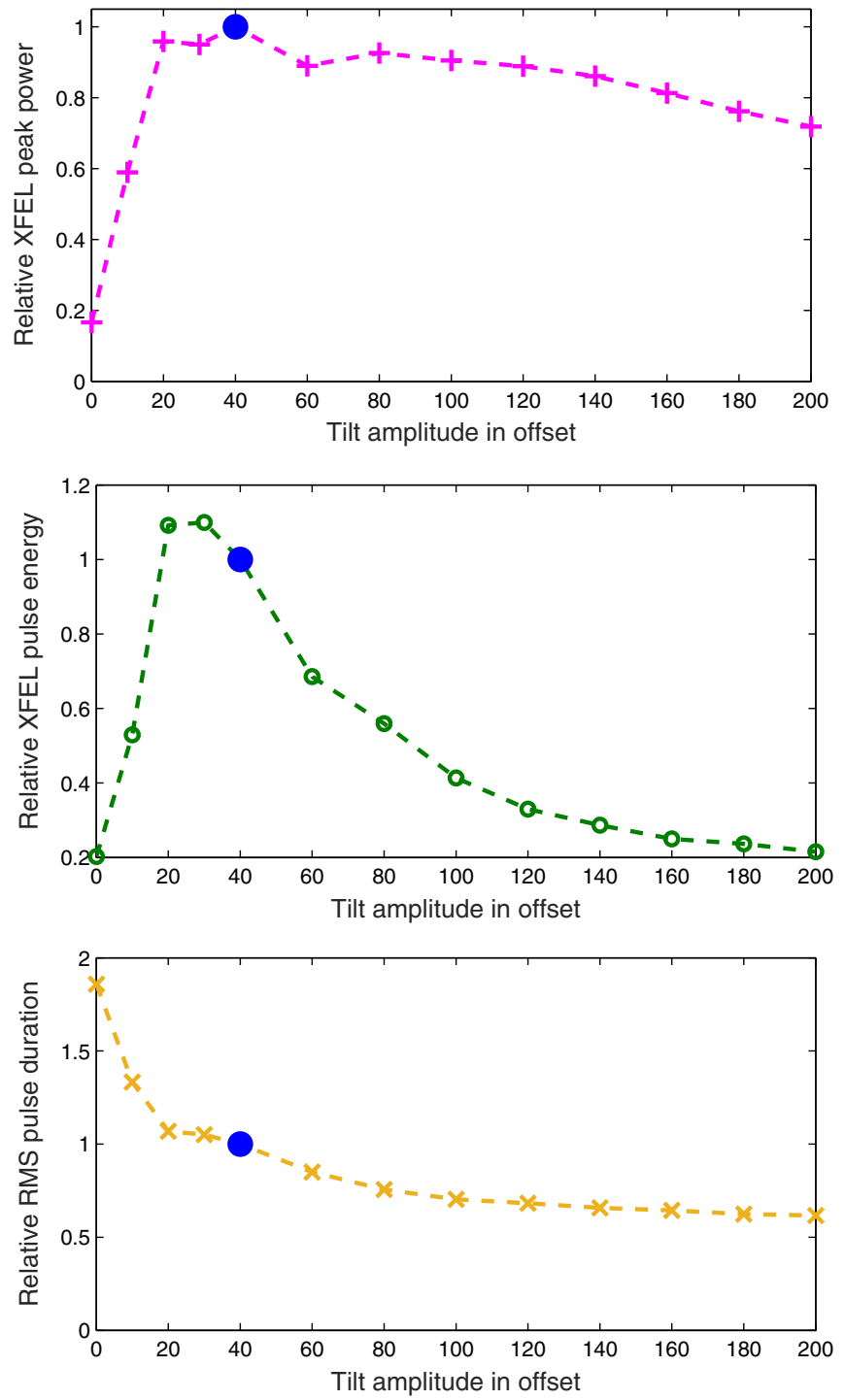

FIG. 6. Relative XFEL peak power (top), XFEL energy (center), and rms pulse duration (bottom) as a function of the initial tilt amplitude in offset. The simulations are done for the SwissFEL parameters with ten undulator sections (total of 15 undulator modules). The reference case (100\% relative power, energy and length) corresponds to the optimum case in terms of XFEL power and is indicated by a blue dot. 
taken in the simulations of the previous subsection), corresponding to the maximum XFEL peak power. The XFEL peak power stays approximately constant around the optimum value and for stronger tilts. As expected, for larger tilts there is a shortening of the pulse length and a decrease of the pulse energy. For initial tilt amplitudes in offset larger than 100, however, there is a continuous decrease of the XFEL power since for such tilt amplitudes there is an increase of the sensitivity of the XFEL pulse power on the trajectory-the XFEL pulse consists of only a few spikes, which require precise alignment. For tilt amplitudes smaller than the optimum one, especially for initial tilt amplitudes in offset lower than 20, the performance of the scheme is diminished since the XFEL pulse is not overlapped with fresh regions of the electron bunch but with degraded ones instead. The scheme is not meaningful for very small or zero tilt amplitudes - in this case a standard undulator beam line configuration without delaying the electron beam would give a better performance. The scheme should always work with GTR around the optimum one or shorter, i.e., with tilt amplitudes around the optimum one or larger.

\section{CONCLUSION}

We have presented a new method to obtain XFEL pulses with significantly higher power and shorter pulse durations than the ones produced at state-of-the-art XFEL facilities. It is based on introducing a transverse tilt to the electron beam, with the result that only a small part of the bunch is able to produce XFEL radiation at each undulator section. In the first undulator section only the tail of the bunch lases, then by suitably delaying the electron beam and correcting its trajectory between certain undulator modules the other regions of the electron bunch can be made to enhance a short XFEL pulse. The method is efficient since all the electrons of the bunch can potentially contribute to the XFEL process. Our proposal is feasible and straightforward to implement in any of the existing or future XFEL facilities: besides the components of a standard facility it only needs additional small chicanes between undulator modules to delay and correct the trajectory of the electrons. The scheme is flexible, since by tuning the tilt one can either maximize the XFEL pulse energy or minimize the pulse length. The method opens the door to new kinds of experiments that require short and high-power XFEL pulses, for instance in the fields of bioimaging and nonlinear optics. We have shown with numerical simulations that the method works: for beam parameters as used for SwissFEL, it is possible to obtain within $70 \mathrm{~m}$ of undulator line $1 \AA$ XFEL radiation with a peak power of about $1 \mathrm{TW}$, a pulse energy of about $0.5 \mathrm{~mJ}$, and an rms pulse duration of about 500 as.

\section{ACKNOWLEDGMENTS}

We thank Referee C of Ref. [26] for stimulating and fruitful discussions. We would like to acknowledge
Thomas Schietinger and Hans Braun for reading the manuscript and helping to improve it, both in language and content.

[1] A. M. Kondratenko and E. L. Saldin, Part. Accel. 10, 207 (1980).

[2] R. Bonifacio, C. Pellegrini, and L. M. Narducci, Opt. Commun. 50, 373 (1984).

[3] J. Feldhaus, E. L. Saldin, J. R. Schneider, E. A. Schneidmiller, and M. V. Yurkov, Opt. Commun. 140, 341 (1997).

[4] E. L. Saldin, E. A. Schneidmiller, Yu. V. Shvyd'ko, and M. V. Yurkov, Nucl. Instrum. Methods Phys. Res., Sect. A 475, 357 (2001).

[5] G. Geloni, V. Kocharyan, and E. Saldin, DESY Report No. 10-053, 2010.

[6] P. Emma et al., Nat. Photonics 6, 693 (2012).

[7] P. Emma et al., Nat. Photonics 4, 641 (2010).

[8] T. Ishikawa et al., Nat. Photonics 6, 540 (2012).

[9] F. Capotondi, M. Dell'Angela, M. Malvestuto, and F. Parmigiani, in Synchrotron Radiation. Basics, Methods and Applications, edited by S. Mobilio, F. Boscherini, and C. Meneghini (Springer, Berlin, 2015), Chap. 30, p. 761.

[10] A. Fratalocchi and G. Ruocco, Phys. Rev. Lett. 106, 105504 (2011).

[11] N. Rohringer et al., Nature (London) 481, 488 (2012).

[12] M. Beye, S. Schreck, F. Sorgenfrei, C. Trabant, N. Pontius, C. Schüßler-Langeheine, W. Wurth, and A. Föhlisch, Nature (London) 501, 191 (2013).

[13] S. Schreck et al., Phys. Rev. Lett. 113, 153002 (2014).

[14] H. Yoneda, Y. Inubushi, M. Yabashi, T. Katayama, T. Ishikawa, H. Ohashi, H. Yumoto, K. Yamauchi, H. Mimura, and H. Kitamura, Nat. Commun. 5, 5080 (2014).

[15] R. Neutze, R. Wouts, D. van der Spoel, E. Weckert, and J. Hajdu, Nature (London) 406, 752 (2000).

[16] P. Emma, K. Bane, M. Cornacchia, Z. Huang, H. Schlarb, G. Stupakov, and D. Walz, Phys. Rev. Lett. 92, 074801 (2004).

[17] S. Reiche, P. Musumeci, C. Pellegrini, and J. B. Rosenzweig, Nucl. Instrum. Methods Phys. Res., Sect. A 593, 45 (2008).

[18] E. L. Saldin, E. A. Schneidmiller, and M. V. Yurkov, Opt. Commun. 239, 161 (2004).

[19] A. A. Zholents and W. M. Fawley, Phys. Rev. Lett. 92, 224801 (2004).

[20] A. A. Zholents, Phys. Rev. ST Accel. Beams 8, 040701 (2005).

[21] A. A. Zholents and G. Penn, Phys. Rev. ST Accel. Beams 8, 050704 (2005).

[22] E. L. Saldin, E. A. Schneidmiller, and M. V. Yurkov, Phys. Rev. ST Accel. Beams 9, 050702 (2006).

[23] A. A. Zholents and M. S. Zolotorev, New J. Phys. 10, 025005 (2008).

[24] D. Xiang, Z. Huang, and G. Stupakov, Phys. Rev. ST Accel. Beams 12, 060701 (2009).

[25] T. Tanaka, Phys. Rev. Lett. 110, 084801 (2013).

[26] E. Prat and S. Reiche, Phys. Rev. Lett. 114, 244801 (2015). 
[27] R. Bonifacio, L. De Salvo Souza, P. Pierini, and N. Piovella, Nucl. Instrum. Methods Phys. Res., Sect. A 296, 358 (1990).

[28] R. Bonifacio, N. Piovella, and B. W. J. McNeil, Phys. Rev. A 44, R3441(R) (1991).

[29] P. Emma and Z. Huang, Nucl. Instrum. Methods Phys. Res., Sect. A 528, 458 (2004).

[30] S. Reiche, P. Emma, and C. Pellegrini, Nucl. Instrum. Methods Phys. Res., Sect. A 507, 426 (2003).

[31] G. A. Loew and O.H. Altenmueller, SLAC Report No. PUB-135, 1965.

[32] B. W. Zotter and S. Kheifets, Impedances and Wakes in High Energy Particle Accelerators (World Scientific, Singapore, 1998).

[33] E. Prat and M. Aiba, Phys. Rev. ST Accel. Beams 17, 032801 (2014).

[34] P. Craievich, Phys. Rev. ST Accel. Beams 13, 034401 (2010).

[35] P. Emma et al., Phys. Rev. Lett. 112, 034801 (2014).
[36] S. Antipov, S. Baturin, C. Jing, M. Fedurin, A. Kanareykin, C. Swinson, P. Schoessow, W. Gai, and A. Zholents, Phys. Rev. Lett. 112, 114801 (2014).

[37] M. W. Guetg, B. Beutner, E. Prat, and S. Reiche, Phys. Rev. ST Accel. Beams 18, 030701 (2015).

[38] S. Reiche, Nucl. Instrum. Methods Phys. Res., Sect. A 429, 243 (1999).

[39] S. Reiche, in Proceedings of the 36th International Free-Electron Laser Conference, Basel, Switzerland, 2014 (JACoW, Geneva, 2015), p. 403.

[40] R. Ganter et al., PSI Report No. 10-04, 2012.

[41] E. Prat, M. Aiba, S. Bettoni, B. Beutner, S. Reiche, and T. Schietinger, Phys. Rev. ST Accel. Beams 17, 104401 (2014).

[42] N. M. Kroll, P. L. Morton, and M. Rosenbluth, IEEE J. Quantum Electron. 17, 1436 (1981).

[43] Y. Jiao, J. Wu, Y. Cai, A. W. Chao, W. M. Fawley, J. Frisch, Z. Huang, H.-D. Nuhn, C. Pellegrini, and S. Reiche, Phys. Rev. ST Accel. Beams 15, 050704 (2012). 